\title{
De novo transcriptome analysis and gene expression profiling of fish scales isolated from Carassius auratus during space flight: Impact of melatonin on gene expression in response to space radiation
}

\author{
YUKIHIRO FURUSAWA ${ }^{1}$, TATSUKI YAMAMOTO ${ }^{2}$, ATSUHIKO HATTORI $^{3}$, NOBUO SUZUKI $^{2}$, \\ JUN HIRAYAMA $^{4}$, TOSHIO SEKIGUCHI ${ }^{2}$ and YOSHIAKI TABUCHI ${ }^{5}$

\begin{abstract}
${ }^{1}$ Department of Liberal Arts and Sciences, Toyama Prefectural University, Toyama 939-0398;
${ }^{2}$ Noto Marine Laboratory, Division of Marine Environmental Studies, Institute of Nature and Environmental Technology, Kanazawa University, Ishikawa 927-0553; ${ }^{3}$ College of Liberal Arts and Sciences, Tokyo Medical and Dental University, Chiba 272-0827; ${ }^{4}$ Department of Clinical Engineering, Faculty of Health Sciences, Komatsu University, Ishikawa 923-0961; ${ }^{5}$ Division of Molecular Genetics Research, Life Science

Research Center, University of Toyama, Toyama 930-0194, Japan
\end{abstract}

Received December 15, 2019; Accepted May 27, 2020

DOI: $10.3892 / \mathrm{mmr} .2020 .11363$

\begin{abstract}
Astronauts are inevitably exposed to two major risks during space flight, microgravity and radiation. Exposure to microgravity has been discovered to lead to rapid and vigorous bone loss due to elevated osteoclastic activity. In addition, long-term exposure to low-dose-rate space radiation was identified to promote DNA damage accumulation that triggered chronic inflammation, resulting in an increased risk for bone marrow suppression and carcinogenesis. In our previous study, melatonin, a hormone known to regulate the sleep-wake cycle, upregulated calcitonin expression levels and downregulated receptor activator of nuclear factor- $\mathrm{\kappa B}$ ligand expression levels, leading to improved osteoclastic activity in a fish scale model. These results indicated that melatonin may represent a potential drug or lead compound for the prevention of bone loss under microgravity conditions. However, it is unclear whether melatonin affects the biological response induced by space radiation. The aim of the present study was to evaluate the effect of melatonin on the expression levels of genes responsive to space radiation. In the present study, to support the previous data regarding de novo transcriptome analysis of goldfish scales, a detailed and improved experimental method (e.g., PCR duplicate removal followed by de novo assembly, global normalization and calculation of statistical significance)
\end{abstract}

Correspondence to: Dr Yukihiro Furusawa, Department of Liberal Arts and Sciences, Toyama Prefectural University, 5180 Kurokawa, Imizu-shi, Toyama 939-0398, Japan

E-mail: furusawa@pu-toyama.ac.jp

Key words: space radiation, transcriptome, de novo assembly, fish scale, gene network, melatonin was applied for the analysis. In addition, the transcriptome data were analyzed via global normalization, functional categorization and gene network construction to determine the impact of melatonin on gene expression levels in irradiated fish scales cultured in space. The results of the present study demonstrated that melatonin treatment counteracted microgravity- and radiation-induced alterations in the expression levels of genes associated with DNA replication, DNA repair, proliferation, cell death and survival. Thus, it was concluded that melatonin may promote cell survival and ensure normal cell proliferation in cells exposed to space radiation.

\section{Introduction}

During space flight, astronauts are exposed to space radiation at $1 \mathrm{mSv}^{-d_{a y}{ }^{-1}}$, a dose rate $\sim 1,000$ times higher than that on the Earth's surface (1). Space radiation consists of constant low-dose, geomagnetic-trapped, high linear energy transfer (LET) particles that are characterized by high relative biological effectiveness (RBE) values (1). Although the radiation dose during space station stays for 90 days is $<4.5$ Gy.30 days ${ }^{-1}$, the lethal dose, chronic high-LET radiation has been suggested to induce more severe DNA damage compared with that of ground radiation (1). Human health should be protected from such high-LET space radiation, thus, as a result, the RBE values of space radiation have been extensively studied in the past decades (2-4).

Nonetheless, the current knowledge of biological effects of space radiation remains limited, as experiments in space cannot be performed as frequently as on the ground. In addition, reproducing the conditions of space flight on the Earth's surface remains difficult. To evaluate the biological effects of space radiation, conducting experiments in actual space are necessary. Previous studies have investigated the biological responses of prokaryotic and eukaryotic organisms 
to radiation during space flight. In particular, D. melanogaster demonstrated a high frequency of DNA mutations after space flight (5). In rat skin and muscle tissue, space flight promoted p53 accumulation, a process known to be catalyzed by radiation-induced DNA damage (6). In addition, Ohnishi et al (7) reported the induction of heat shock protein $(H S P) 72$, a gene responsive to stresses such as radiation and heat, in the muscle, skin and spleen of goldfish, a non-model vertebrate, under space flight.

Melatonin is a hormone involved in the regulation of the sleep-wake cycle, demonstrating protective properties against ionizing radiation (8). The pineal gland is a major source of melatonin; however, melatonin is also synthesized in numerous extrapineal tissues, including the skin, bone marrow and gastrointestinal tract, leading to high concentrations of melatonin in peripheral tissues (9). Supporting the local production of melatonin, $\mathrm{N}$-acetylserotonine, a direct precursor of melatonin, is also expressed in peripheral tissues (10). Although melatonin levels are regulated by metabolism in the liver or peripheral organs (e.g. kidney and uterus), the metabolites (e.g. 6-hydroxymelatonin and 5-methoxytryptamine) and melatonin function in both a receptor-dependent and -independent manner $(11,12)$. The receptor-independent mechanism was identified to involve melatonin serving as a mitochondrial-targeted antioxidant that protected DNA and cells from damage (13). Melatonin has also been discovered to attenuate the cytotoxicity of ionizing radiation, including DNA damage and apoptosis, without exerting any cytotoxic effect on normal organ function (8). Therefore, melatonin may represent a promising compound to alleviate the side effects of radiation, (e.g., as a radioprotector during clinical radiotherapy).

Advances in next-generation sequencing (NGS) and bioinformatics have permitted the determination of genome-wide expression profiles, as well as their associated biological functions and gene networks, even in non-model organisms with no established databases (14-16). Using NGS in goldfish, our previous study demonstrated that melatonin suppressed the osteoclastic activity induced by microgravity, indicating that melatonin may be a potential drug to treat space flight-induced bone loss (17). However, it remains unknown whether melatonin has an impact on biological responses induced by space radiation.

The present study aimed to examine the effect of radiation during space flight on gene expression levels in goldfish scales, a model that was previously used to examine the biological responses to various stimuli (17). In addition, to determine the potential of melatonin as a radioprotective drug during space flight, RNA-sequencing (RNA-seq) analysis with subsequent de novo transcriptome assembly and computational gene expression analysis were also performed.

\section{Materials and methods}

Fish scale isolation and culture. Equal numbers of male and female immature Carassius auratus (goldfish) specimens, purchased from Higashiyama Fish Farm, were grown until the body size reached $10-12 \mathrm{~cm}$. A total of 16 goldfish were kept in an aquarium at $26^{\circ} \mathrm{C}$ under a $12 \mathrm{~h}$ light/dark cycle and fed every morning. Thereafter, the scales were collected from the goldfish under anesthesia (64 scales were collected from each goldfish). For anesthesia, the goldfish were placed into a $300 \mathrm{mg} / 1(0.03 \%)$ ethyl 3-aminobenzoate and methane sulfonic acid salt solution (MS-222; Nacalai Tesque, Inc.) neutralized with $0.03 \%$ sodium bicarbonate. The fish scales were isolated and cultured during space flight as previously described (17). Briefly, the isolated fish scales were regenerated for 14 days and packaged into a culture chamber for space flight and ground $(\mathrm{G})$ experiments. The fish scales were stored at $4^{\circ} \mathrm{C}$ prior to microgravity culture and incubated for $86 \mathrm{~h}$ at $22^{\circ} \mathrm{C}$ under in-flight microgravity $(\mathrm{F}-\mu \mathrm{G})$ or in-flight artificial 1 gravity (F-1G; without microgravity) by centrifugation at $1 \mathrm{x} \mathrm{g}$ and $22^{\circ} \mathrm{C}$ as previously reported (18). Fish scales under $\mathrm{F}-\mu \mathrm{G}$ and $\mathrm{G}$ conditions (F-1G excluded due to limited sample numbers) were also treated with Leibovitz's L-15 medium (Invitrogen; Thermo Fisher Scientific, Inc.) containing $0.01 \%$ DMSO and $1 \mu \mathrm{M}$ melatonin (Sigma-Aldrich; Merck KGaA). $\mathrm{F}-\mu \mathrm{G}+$ melatonin and $\mathrm{G}+$ melatonin, respectively) and were maintained at $4^{\circ} \mathrm{C}$ just before space flight (5). All procedures in the present study were approved by the Institutional Animal Care and Use Committee of Kanazawa University (Noto-cho, Japan) and performed in accordance with the guidelines for animal experiments provided by the Ethics Committee of Kanazawa University.

RNA isolation and sequencing. Total RNA was isolated from fish scales using the RNeasy Mini kit (Qiagen, Inc.), according to the manufacturer's protocol. RNA quality was analyzed using a Bioanalyzer 2100 instrument and the RNA6000 Nano LabChip ${ }^{\circledR}$ kit (both Agilent Technologies, Inc.). RNA samples with RNA integrity number values of $>9.0$ were used for library construction. RNA concentration was determined by Nanodrop spectrometer (Thermo Fisher Scientific, Inc.) and Bioanalyzer followed by library construction. RNA-seq libraries for paired-end reads $100 \mathrm{bp}$ in length from biologically triplicated samples were constructed using TruSeq RNA Sample Prep kit v2 (cat. no. RS-122; Illumina, Inc.). 10-20 pM pooled libraries were sequenced using $\mathrm{HiSeq}^{\mathrm{TM}}$ 2000 (Illumina, Inc.). The raw sequence reads were deposited at the DNA Data Bank of Japan (DDBJ; https://ddbj. nig.ac.jp/DRASearch/submission?acc=DRA008502) under the DDBJ Sequence Read Archive (DRA) accession no. DRA009118.

Quality control check and trimming of reads. Quality check of raw read sequences was performed using FastQC (v0.11.9; bioinformatics.babraham.ac.uk/projects/fastqc). Adaptors and short, low-quality reads $(\mathrm{Q}<20$ and $<36 \mathrm{bp})$ were trimmed using Trimmomatic v0.39 (usadellab.org/cms/?page=trimmomatic) with default parameters (19). The number of raw and processed reads are presented in Table I.

De novo transcriptome assembly and read mapping. Following PCR duplicate removal using filterPCRdupl v1.01 (20), the de novo transcriptome assembly of processed reads was performed using Trinity (version r2012-10-05) with default parameters to generate a reference sequence of transcripts (21). Trinity was equipped with Management and Analysis System for Enormous Reads (Maser; cell-innovation. nig.ac.jp/index_en.html), a graphical user interface-based tool for NGS analysis, which was used to run Trinity for 
Table I. Summary of the number of raw reads, processed reads and mapping rate of transcriptome data.

\begin{tabular}{|c|c|c|c|c|}
\hline Treatment & Sample ID & Raw reads, count & Processed reads, count & Mapping rate, $\%$ \\
\hline $\mathrm{F}-1 \mathrm{G}$ & S001 & $21,382,556$ & $20,946,002$ & 92.46 \\
\hline $\mathrm{F}-1 \mathrm{G}$ & S002 & $21,506,294$ & $21,104,591$ & 92.27 \\
\hline $\mathrm{F}-1 \mathrm{G}$ & $\mathrm{S} 003$ & $28,155,809$ & $27,650,984$ & 92.39 \\
\hline $\mathrm{F}-\mu \mathrm{G}$ & S004 & $21,156,047$ & $20,789,017$ & 92.34 \\
\hline $\mathrm{F}-\mu \mathrm{G}$ & S005 & $21,655,997$ & $21,255,721$ & 92.10 \\
\hline $\mathrm{F}-\mu \mathrm{G}$ & S006 & $21,139,783$ & $20,768,132$ & 92.23 \\
\hline $\mathrm{G}$ & S007 & $25,132,537$ & $24,638,896$ & 91.50 \\
\hline $\mathrm{G}$ & S008 & $24,131,108$ & $23,677,305$ & 91.47 \\
\hline $\mathrm{G}$ & S009 & $20,658,328$ & $20,215,123$ & 92.18 \\
\hline $\mathrm{F}-\mu \mathrm{G}+$ melatonin & S010 & $23,724,796$ & $23,497,407$ & 91.89 \\
\hline $\mathrm{F}-\mu \mathrm{G}+$ melatonin & S011 & $22,484,040$ & $22,260,350$ & 91.68 \\
\hline $\mathrm{F}-\mu \mathrm{G}+$ melatonin & S012 & $22,737,402$ & $22,469,979$ & 91.66 \\
\hline $\mathrm{G}+$ melatonin & S013 & $25,120,774$ & $24,599,748$ & 91.39 \\
\hline $\mathrm{G}+$ melatonin & S014 & $24,027,734$ & $23,539,894$ & 91.93 \\
\hline $\mathrm{G}+$ melatonin & S015 & $24,379,991$ & $23,958,109$ & 92.25 \\
\hline
\end{tabular}

G, ground samples; F- $\mu \mathrm{G}$, space flight samples with microgravity; F-1G, space flight samples with $1 \mathrm{G}$.

de novo assembly. PCR duplicates were filtered to remove the sequence noise and decrease the number of transcript candidates. Sequence reads including PCR duplicates were mapped to the reference using Bowtie2 v2.2.5 with strict parameters (-D 20-R 3-N 0-L 20-i S,1,0.50) (22) to increase the rate of unique mapping. The mapping rates are presented in Table I.

Transcriptome annotation using Blastx. Blastx 2.2.26+ (blast. ncbi.nlm.nih.gov/Blast.cgi?LINK_LOC=blasthome\&PAGE_ TYPE=BlastSearch\&PROGRAM=blastx) equipped with Maser was used to annotate the reference sequences using default parameters and an e-value cutoff of $<10^{-10}$. The resulting top hit genes were listed, and a total of 84,748 from 175,357 transcript candidates were annotated. All UniProt accession numbers, subject IDs and sequence identities are provided in Table SI.

Transcript quantification and identification of differentially expressed genes (DEGs). Estimation of relative RNA expression levels from mapped reads and principal component analysis (PCA) was performed using Strand NGS v3.3 (strand-ngs.com) with the Trimmed Mean of M value (TMM) normalization method (23). TMM normalization is more reliable than using fragments per kilobase of exon per million reads mapped (12). DEGs were identified using one-way ANOVA followed by post-hoc Tukey's test and multiple testing Benjamini-Hochberg correction to calculate false discovery rate and adjust P-value. Genes were considered DEGS where q-value (adjusted P-value) $<0.05$ and fold change $>1.3$ or $<-1.3$ for up- and downregulated genes, respectively. Venn diagrams were drawn using Strang NGS.

Analysis of molecular functions and gene networks. To determine the molecular functions of the DEGs and gene networks involved, expression data were analyzed using
Ingenuity Pathway Analysis (IPA) tools (Qiagen, Inc.). In order to import DEGs, the transcript candidates were converted to Gene IDs using The Database for Annotation, Visualization and Integrated Discovery 6.8 (24). IPA is a web-delivered application that enables the identification, visualization and exploration of molecular interaction networks in gene expression data. The top five molecular functions were identified, and their associated gene networks were visualized to obtain information on the interactions of upregulated and downregulated genes during melatonin treatment. The network is displayed graphically as nodes (genes) and edges (biological associations). Heatmaps of the expression values of genes on the identified gene networks were constructed via Microsoft Excel 2019 software (Microsoft Corporation).

\section{Results}

Identification of differentially expressed transcripts responsive to space radiation and melatonin treatment. The effect of melatonin on gene expression alterations in response to microgravity was determined in a previous study by comparing fish scale DEGs on ground and in space flight in the presence or absence of microgravity (17). In the present study, to identify the candidate transcripts responsive to space irradiation, de novo transcriptome analysis of fish scales was performed, focusing on upregulated and downregulated genes during space flight between fish scales with $1 \mathrm{G}$ or microgravity (F-1G and $\mathrm{F}-\mu \mathrm{G}$; Fig. 1). After quantification and TMM normalization of mapped reads, principal component analysis (PCA) was performed on the expression data (Fig. 2). The expression pattern of transcripts in the $G$ group (low-radiation control) was distinct compared with melatonin treatment on ground $(\mathrm{G}+$ melatonin) or in space flight $(\mathrm{F}-\mu \mathrm{G}+$ melatonin). Moreover, the untreated space flight groups F-1G and $\mathrm{F}-\mu \mathrm{G}$ displayed similar expression patterns to the $\mathrm{G}$ group, which were distinct from the melatonin-treated groups. 


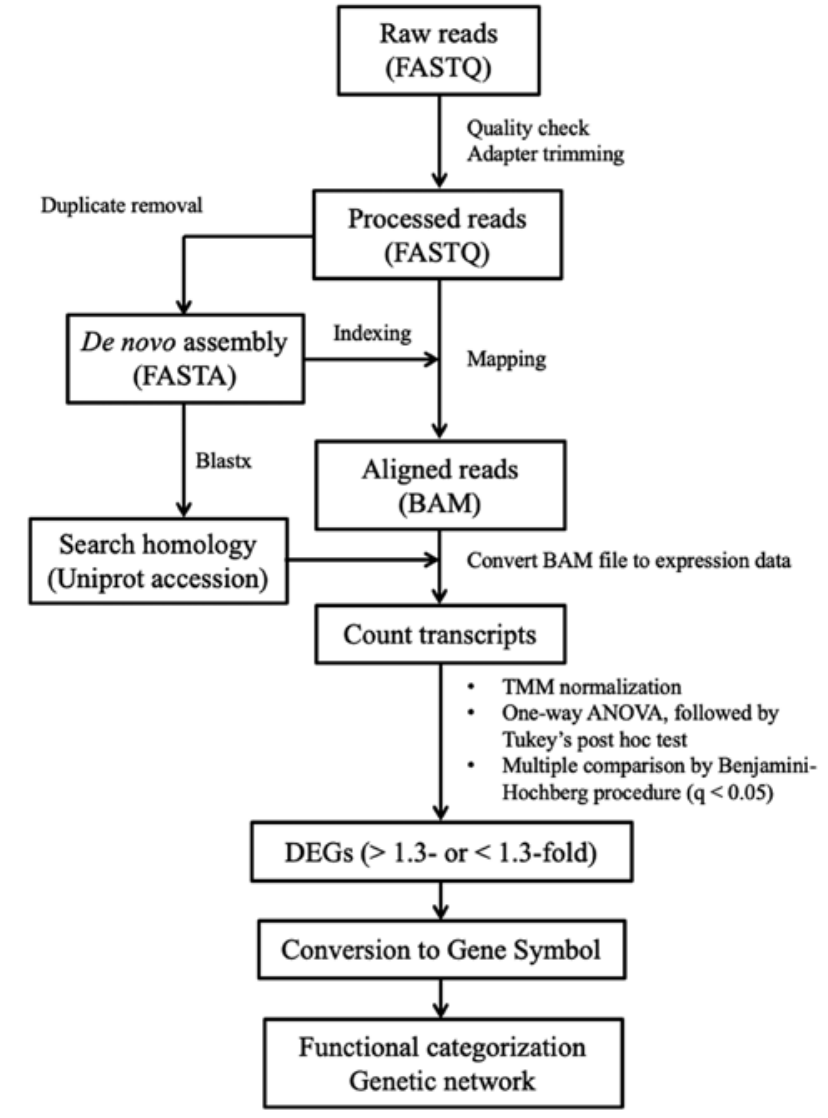

Figure 1. Workflow of de novo transcriptome analysis in goldfish scales. DEGs, differentially expressed genes; TMM, Trimmed Mean of M value.

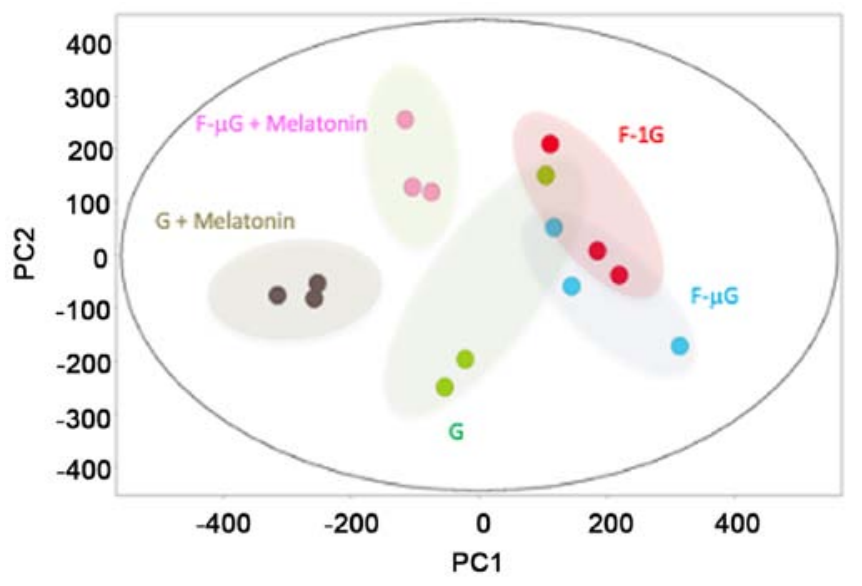

Figure 2.PCA of the comprehensive gene expression data.PCA was performed on data from the $\mathrm{G}, \mathrm{F}-\mu \mathrm{G}, \mathrm{F}-1 \mathrm{G}, \mathrm{G}+$ melatonin and $\mathrm{F}-\mu \mathrm{G}+$ melatonin groups. Fish scales isolated from Carassius auratus were cultured on ground condition or space flight in the presence or absence of microgravity/melatonin until freezing, followed by RNA extraction and transcriptome analysis. The data represents a single sequencing experiment of biological triplicates PCA, principal component analysis; G, ground samples; F- $\mu \mathrm{G}$, space flight samples in the presence of microgravity; F-1G space flight samples in the absence of microgravity.

Among differentially expressed 2,446 transcripts between the ground and space flight groups, 1,409 were upregulated and 1,037 were downregulated during space flight, regardless of the presence of microgravity (Fig. 3A). The impact of melatonin on DEGs was also assessed by comparing the melatonin-treated samples. Among the 1,409 upregulated and 1,037 downregulated transcripts, the expression of 846 and 714 transcripts were altered by melatonin treatment (Fig. 3B, left and right, respectively). Among them, melatonin reversed the expression patterns of 774 and 600 transcripts, respectively, that were altered by space radiation (Fig. 3B). Furthermore, among 774 and 600 transcripts responsive to melatonin, 576 and 219 transcripts had UniProt accession numbers assigned by Blastx (Table SI). Among the transcripts with UniProt accession numbers, 566 upregulated and 208 downregulated transcripts responsive to space radiation had high sequence homology with genes identified in Homo sapiens, Mus musculus, Danio rerio and other species (Tables SII and SIII). The annotated transcripts were designated as DEGs for subsequent computational analysis.

Functional analysis of genes responsive to space radiation and melatonin. The molecular and cellular functions and corresponding genetic networks of the DEGs were subsequently identified in order to assess the impact of melatonin on gene expression levels altered by space radiation. Analysis of canonical pathways based on the IPA indicated that melatonin reduced the number of DEGs associated with 'Unfolded Protein Response' consisting of HSP families that are upregulated under space conditions (7), 'NRF-2-mediated Oxidative Stress Response' consisting of superoxide dismutase 2, as well as 'NER Pathway' and 'DNA Double-Strand Break Repair by Non-Homologous End Joining', consisting of DNA ligase (LIG)4, excision repair cross-complementing (ERCC2) and protein kinase, DNA-activated, catalytic subunit (PRKDC (Table II).

Functional analysis also suggested that the genes downregulated by melatonin were involved in 'Cell Death and Survival' and 'DNA Replication, Recombination, and Repair' (Fig. 4). Genes upregulated by melatonin were also associated with 'Cellular Development', 'Cell Death and Survival' and 'Cellular Growth and Proliferation' comprising Bcl family members and cyclin-dependent kinase 1B (CDKN1B).

Gene networks responsive to space radiation and melatonin. Gene network analysis by IPA was performed to elucidate the interactions between DEGs responsive to space radiation (Fig. 5).

An upregulated gene network containing 16 genes was identified, which included HSP family genes such as DNA J heat shock protein member (DNAJ)A1, DNAJB1 and HSPD1. The network also contained PRKDC, mediator of DNA damage checkpoint 1 (MDC1), LIG3, Rad18 E3 ubiquitin ligase (RAD18), DNA polymerase $\kappa(\mathrm{POLK})$ and ERCC2, which are genes involved in DNA double-strand break (DSB) repair and nucleotide excision repair $(25,26)$ (Fig. $5 \mathrm{~A}$ and C). In addition, apoptosis-inducing factor 1 , mitochondria-associated (AIFM1) was discovered to be upregulated by space radiation. AIFM1 encodes apoptosis-inducing factor 1, which is released from the mitochondria and involved in caspase-independent DNA fragmentation during apoptosis (27). Checkpoint kinase 2 (CHEK2) was also upregulated; this gene encodes CHK2, a kinase involved in p53-dependent apoptosis induced by ionizing radiation (28). Marker of proliferation Ki67 (MKI67) and proliferating cell nuclear antigen (PCNA) were also identified in the upregulated network. 
A

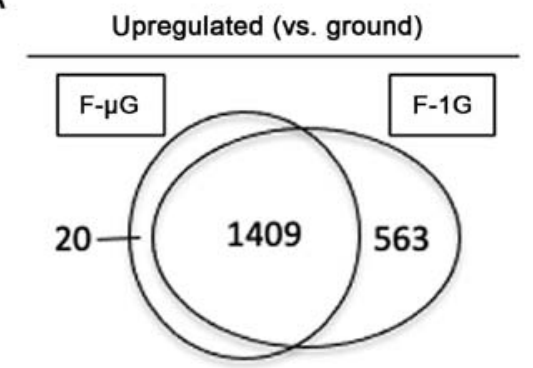

Downregulated (vs. ground)

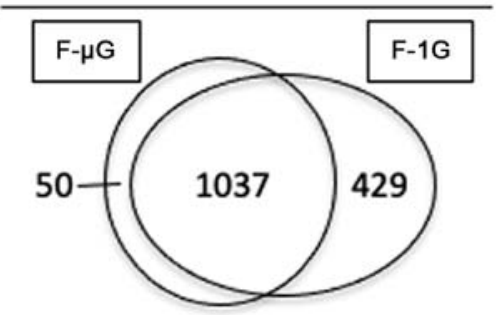

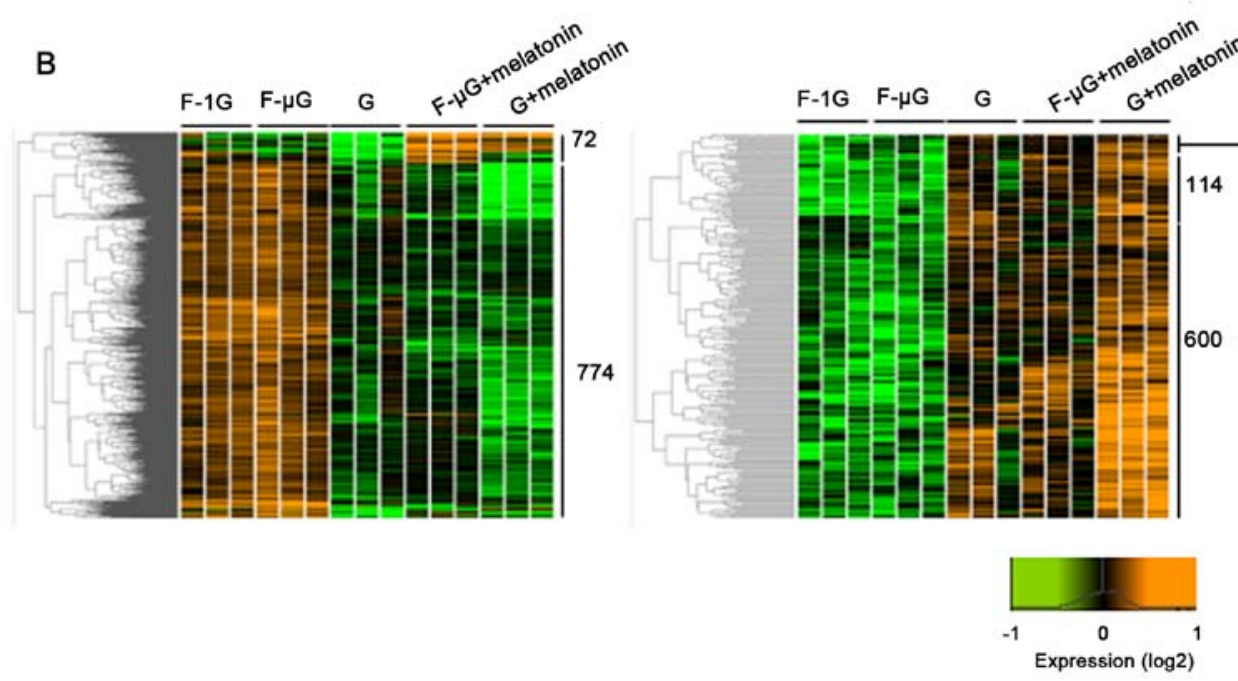

Figure 3. Venn diagrams and hierarchical clustering of identified differentially expressed transcripts in goldfish scales. (A) Venn diagrams of upregulated and downregulated transcripts between the G group and space flight samples F- $\mu \mathrm{G}$ or F-1G. (B) Heatmaps of the differentially expressed transcripts identified from $\mathrm{G}, \mathrm{F}-\mu \mathrm{G}, \mathrm{F}-1 \mathrm{G}, \mathrm{G}+$ melatonin and $\mathrm{F}-\mu \mathrm{G}+$ melatonin groups. The color spectrum (green to orange) represents the $\log 2$ expression values scaled between- 0.66 and 0.66 (low to high expression). G, ground samples; F- $\mu \mathrm{G}$, space flight samples in the presence of microgravity; F-1G space flight samples in the absence of microgravity.

The identified downregulated gene network contained 15 genes, including anti-apoptotic Bcl family genes such as BCL2, BCL3 and BCL11B, that were downregulated by space radiation (Fig. 5B and C). CDKN1B, which is involved in cell-cycle progression, was also downregulated by space radiation (29). Several transcription factors, such as Forkhead box O1 (FOXO1), runt-related transcription factor 2 (RUNX2), myocyte enhancer factor 2C (MEF2C) and nuclear receptor corepressor 1 (NCOR1) were also identified in the downregulated gene network. In both networks, the treatment with melatonin recovered the expression levels of DEGs altered by space radiation but did not markedly affect gene expression in the control groups (Fig. 5C), indicating that melatonin may reverse the changes in gene expression levels induced by space radiation without affecting constitutive gene expression.

\section{Discussion}

The influence of low-dose rate radiation on human health during space flight has been investigated in actual space conditions. For instance, previous studies demonstrated that HSP-encoding genes were upregulated by ionizing radiation, not only on the ground but also during space flight in mammalian and goldfish spleens $(7,30)$. Similarly, the upregulation of several HSP-encoding genes, such as DNAJA1, DNAJB1 and HSPD1, in fish scales exposed to space radiation was also observed in the present study, both in the absence and presence of microgravity. Gene network analysis also suggested that the upregulated HSP-coding genes may interact with other genes responsive to space radiation. Among them, PRKDC is known to serve a pivotal role in non-homologous end joining (NHEJ) of double-stranded DNA (31). DSBs, induced by direct ionization of DNA or hydroxyl radical attacks, are the most harmful form of DNA damage for cells, since even a single unrepaired DSB site can cause cell death (32). In the absence of sister chromatids in $\mathrm{G}_{0} / \mathrm{G}_{1}$-phase cells, NHEJ is the major pathway for DSB repair (32). Although the upregulation of $P R K D C$ has not yet been demonstrated during space flight, it may be a potential protective response to chronic low-dose high LET space radiation. In the presence of sister chromatids in $\mathrm{S}$ and $\mathrm{G}_{2} / \mathrm{M}$-phase cells, the homologous recombination (HR) pathway that is highly conserved among species, can lead to accurate DSB repair (31). MDC1, a component of the MDC1/RAD50 DSB repair protein/nibrin complex (33), has been identified to bind to damaged sites and recruit DNA damage sensor proteins, such as ataxia telangiectasia, and DNA repair proteins, including tumor suppressor 53 binding protein 1 to promote HR (34). In addition, MDC1 was discovered to activate CHK2 in the mammalian DNA damage response pathway (35). Protein-protein interactions between MDC1 and CHK2 have not yet been reported in goldfish. However, 
Table II. List of identified IPA canonical pathways and associated genes that were differentially expressed in response to space radiation and melatonin treatment.

\begin{tabular}{lcl}
\hline Ingenuity Canonical Pathways & $-\log ($ P-value $)$ & \multicolumn{1}{c}{ Gene } \\
\hline Protein Ubiquitination Pathway & 8.22 & ANAPC4, DNAJA1, DNAJB1, DNAJB9, DNAJC3, HSP90AA1, HSP90B1, \\
& & $\begin{array}{l}\text { HSPA4, HSPD1, HSPE1, PSMA3, PSMC1, PSMC2, PSMC4, PSMC5, } \\
\text { PSMD10, PSMD6, PSMD8, UBE2D4, UCHL1, USP22 }\end{array}$ \\
NRF2-Mediated Oxidative & 2.91 & $\begin{array}{l}\text { CBR1, CLPP, DNAJA1, DNAJA4, DNAJB1, DNAJB9, DNAJC3, GSR, } \\
\text { PPIB, SOD2 }\end{array}$ \\
$\begin{array}{l}\text { NER Pathway } \\
\text { Role of BRCA1 in DNA }\end{array}$ & 2.76 & $\begin{array}{l}\text { ERCC2, GTF2H5, LIG3, POLE3, POLK, RFC5, TCEA1 } \\
\text { BRIP1, CHEK2, MDC1, RFC5, SMARCA4, SMARCD1 }\end{array}$
\end{tabular}

Damage Response

DNA Double-Strand Break Repair $\quad 1.63 \quad$ LIG3, PRKDC

by Non-Homologous End Joining

Genes associated with each canonical pathway were selected via IPA. P-values represent the whole pathway. IPA, Ingenuity Pathway Analysis; ANAPC4, anaphase promoting complex subunit; DNAJA1, DnaJ homolog subfamily A member 1; DNAJB1, DnaJ homolog subfamily B member 1; DNAJB9, DnaJ homolog subfamily B member 9; DNAJC3, DnaJ homolog subfamily C member 3; HSP90AA1, heat shock protein $90 \alpha$ family class A member 1; HSP90B1, heat shock protein $90 \beta$ family member 1; HSPA4, heat shock 70 kDa protein 4; HSPD1, heat shock protein family D member 1; HSPE1, heat shock protein family E member 1; PSMA3, proteasome 20S subunit $\alpha$ 3; PSMC1, proteasome 26S subunit ATPase 1; PSMC2, proteasome 26S subunit ATPase 2; PSMC4, proteasome 26S subunit ATPase 4; PSMC5, proteasome 26S subunit ATPase 5; PSMC10, proteasome 26S subunit ATPase 10; PSMC6, proteasome 26S subunit ATPase 6; PSMC8, proteasome 26S subunit ATPase 8; UBE2D4, ubiquitin-conjugating enzyme E2 D4; UCHL1, ubiquitin C-terminal hydrolase L1; USP22, ubiquitin-specific peptidase 22; CBR1, carbonyl reductase 1; CLPP, caseinolytic mitochondrial matrix peptidase proteolytic subunit; GSR, glutathione disulfide reductase; SOD2, superoxide dismutase 2; ERCC2, excision repair cross-complementing 2; GTF2H5, general transcription factor IIH subunit 5; Lig3, DNA ligase 3; POLE3, DNA polymerase $\varepsilon$ 3; POLK, DNA polymerase $\kappa$; RFC5, replication factor C subunit 5; TCEA1, transcription elongation factor A1; BRIP1, BRCA1-interacting protein C-terminal helicase 1; CHEK2, checkpoint kinase 2; MDC1, mediator of DNA damage checkpoint 1; SMARCA4, SWIF/SNF-related, matrix-associated, actin-dependent regulator of chromatin, subfamily A, member 4; SMARCD1, SWIF/SNF-related, matrix-associated, actin-dependent regulator of Chromatin, subfamily D, member 1; PRKDC, protein kinase, DNA-activated, catalytic subunit.

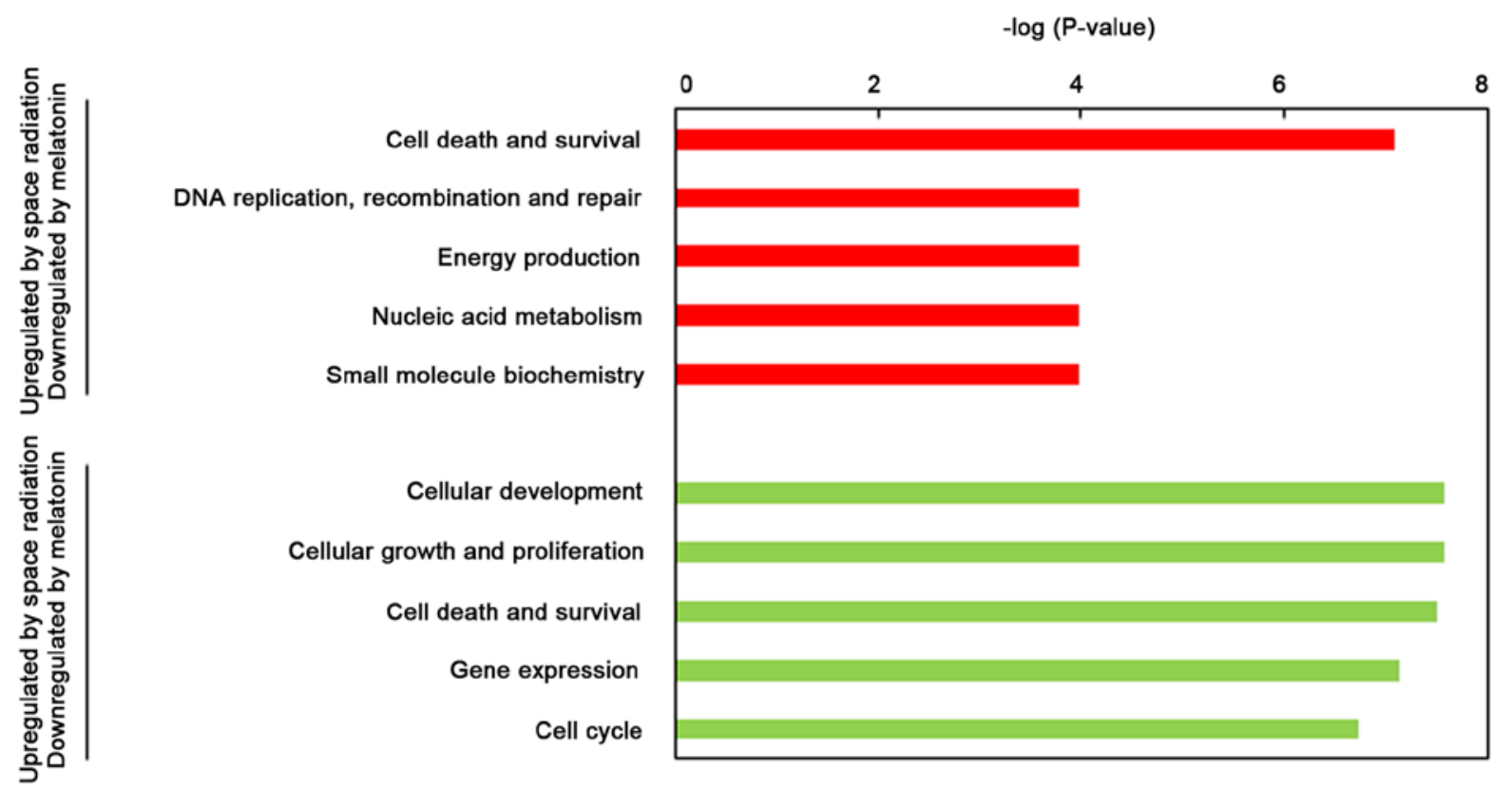

Figure 4. Molecular and cellular function analysis of genes responsive to melatonin. Top five molecular and cellular functions of upregulated and downregulated genes based on P-values calculated by Ingenuity Pathway Analysis.

the simultaneous upregulation of these genes in the present study suggested the possibility of coupled activation in their corresponding proteins. In addition, RAD18 and POLK may also potentially interact with CHK2. RAD18 is involved in translesion synthesis, which prevents replication fork stalling at damaged sites by interacting with POLK (36). CHK2 and 

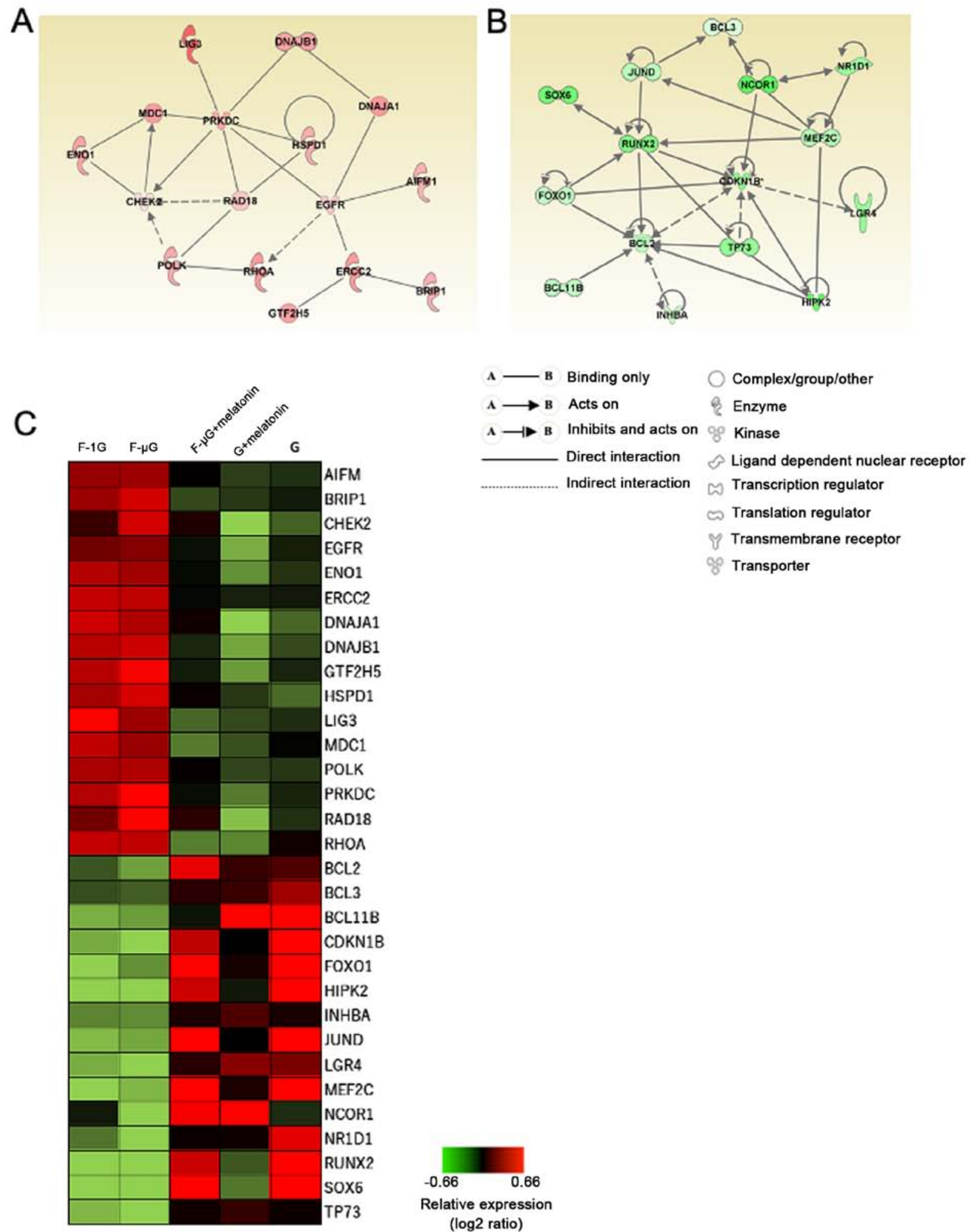

Figure 5. Gene networks identified using differentially expressed gene analysis. (A) Upregulated and (B) downregulated genes following melatonin treatment were analyzed using Ingenuity Pathway Analysis tools. Red nodes represent upregulated genes, while the green nodes are for downregulated genes. Solid lines and dashed lines indicate direct and indirect interactions between molecules, respectively. (C) Heatmap of genes in the identified networks based on relative expression values. The green to red color spectrum represents the $\log 2$ ratios scaled between- 0.66 and 0.66 (low to high expression). $\mathrm{G}$, ground samples; $\mathrm{F}-\mu \mathrm{G}$, space flight samples with microgravity; F-1G space flight samples with 1G. The expression value and gene names are also shown in Table SII. EGFR, epidermal growth factor receptor; ENO1, enolase 1; RHOA, Ras homologue family member A; HIPK2, homeodomain-interacting protein kinase 2; INHBA, inhibin subunit $\beta$ A; LGR4, leucine-rich repeat-containing G protein-coupled receptor 4; Sox6, SRY-box transcription factor 6.

RAD18 have been previously identified to cooperatively maintain genome stability during frequent lymphomagenesis in double knockout mice (37).
In the present study, melatonin treatment counteracted the radiation-induced upregulation of DNA repair genes. A previous study demonstrated that melatonin has the 
potential to scavenge free radicals, such as reactive oxygen and nitrogen species (38). Although the detailed mechanism on how melatonin affects upregulated DNA repair genes in response to space radiation has yet to be determined, one possible explanation is that melatonin treatment may serve as a radical scavenger that ameliorates DNA damage (8), thus resulting in moderate DNA repair.

Previous studies have reported that the Bcl-2 anti-apoptotic protein was downregulated by ionizing radiation, thereby promoting apoptosis (39). Melatonin has also been reported to protect cells from radiation-induced apoptosis by recovering Bcl-2 expression (40). Similarly, in the present study, the expression of BCL-2 in goldfish scales was downregulated by space radiation, which was subsequently recovered following melatonin treatment. Furthermore, other anti-apoptotic Bcl family genes, such as BCL3 and BCL11B $(41,42)$, were also downregulated by space radiation. The attenuation of BCL2 expression may be explained by the observed downregulation of upstream transcription factors that bind the BCL2 promoter and elicit BCL2 expression, such as FOXO1 and RUNX2 $(43,44)$. The expression of BCL family genes was also restored by melatonin treatment, suggesting that melatonin may antagonize the anti-apoptotic response elicited by space radiation.

The identified network contained BCL2-interacting genes such as CDKN1B, RUNX2, FOXO1 and NCOR1. CDKN1B codes for $\mathrm{p} 27^{\mathrm{Kip} 1}$, a CDK inhibitor that promotes $\mathrm{G}_{1}$ cell cycle arrest by inhibiting CDK4 (45). In general, $C D K N 1 B$ has been found to be upregulated following DNA damage induced by acute high-dose ionizing radiation (46). However, in the present study, CDKN1B expression was downregulated by chronic low-dose space radiation. Interestingly, in the present study, MKI67 and PCNA, which are well-studied proliferation markers (47), were upregulated in the presence of space radiation. Upregulation of proliferative genes may be explained by the hormesis effect evoked by low-dose radiation (48). The molecular mechanism underlying hormesis effect remains unknown. However, the regulation of CDKN1B expression by transcription factors RUNX2, FOXO1 and NCOR1 may be one possible explanation (49-51). MEF2C may also be a possible regulator, since MEF2C regulates RUNX2 expression by directly binding to its enhancer region (52). JunD proto-oncogene, AP-1 transcription factor subunit is also a candidate regulator because its stable expression enhances the expression of RUNX2 (53).

Previous studies have demonstrated the radioprotective effect of melatonin in a receptor-independent manner (54), which was at least partly mediated by radical scavenging and the activation of DNA repair machinery $(55,56)$. The present study demonstrated that melatonin suppressed the expression levels of DNA repair genes that were upregulated by radiation-induced DNA damage, supporting the radioprotective effect of melatonin on DNA at the gene expression level, and provided valuable information regarding the possible application of melatonin as a radioprotector during space flight. Melatonin has been suggested to exert a radioprotective effect either directly or indirectly through its metabolite, as melatonin can be rapidly metabolized in peripheral tissue $(57,58)$.
In conclusion, the findings of the present study suggested that melatonin treatment may counteract the expression of anti-apoptotic and proliferative genes attenuated by space radiation. In addition, melatonin also counteracts the expression levels of genes associated with DNA damage that were upregulated by space radiation. Thus, melatonin may promote cell survival and normal cell proliferation during space flight. Whether and how melatonin affects the gene response of mammalian cells to space radiation remains to be determined. Furthermore, comparisons between transcriptome and proteome data should be performed to confirm whether the genes responsive to space radiation are functional in goldfish scales.

\section{Acknowledgements}

The authors wish to acknowledge the Japan Manned Space System and Chiyoda Corporation for their technical support, development of equipment and instruction on how to use the equipment. The authors would also like to thank the Japanese Astronaut Mr Soichi Noguchi for performing experiments at the International Space Station.

\section{Funding}

The present study was supported partly by grants to NS (Japan Aerospace Exploration Agency and Kanazawa University Chozen Project), to AH (The Cooperative Research Program of The Institute of Nature and Environmental Technology, Kanazawa University; grant no. 17018) and to JH (The Cooperative Research Program of The Institute of Nature and Environmental Technology, Kanazawa University; grant no. 19024).

\section{Availability of data and materials}

The datasets are available in the DNA DataBank Japan repository, https://ddbj.nig.ac.jp.

\section{Authors' contributions}

YF and YT analyzed and interpreted the data. YF wrote the manuscript. AH and NS designed study. TY, JH and TS supported data analysis and performed validation. All authors read and approved the final manuscript.

\section{Ethics approval and consent to participate}

All procedures in the present study were approved by the Institutional Animal Care and Use Committee of Kanazawa University (Noto-cho, Japan) and performed in accordance with the guidelines for animal experiments provided by the Ethics Committee of Kanazawa University.

\section{Patient consent for publication}

Not applicable.

\section{Competing interests}

The authors declare that they have no competing interests. 


\section{References}

1. Ohnishi $\mathrm{K}$ and Ohnishi T: The biological effects of space radiation during long stays in space. Biol Sci Space 18: 201-205, 2004

2. Ohnishi T and Nagaoka S: Emphasis of biological research for space radiation. Biol Sci Space 12: 5-13, 1998 (In Japanese).

3. Takahashi A and Ohnishi T: Space radiation biology. Biol Sci Space 15: 40-46, 2001 (In Japanese).

4. Hellweg CE, Dilruba S, Adrian A, Feles S, Schmitz C, Berger T, Przybyla B, Briganti L, Franz M, Segerer J, et al: Space experiment 'Cellular Responses to Radiation in Space (CellRad)' Hardware and biological system tests. Life Sci Space Res (Amst) 7: 73-89, 2015.

5. Ikenaga M, Yoshikawa I, Kojo M, Ayaki T, Ryo H, Ishizaki K, Kato T, Yamamoto $\mathrm{H}$ and Hara R: Mutations induced in Drosophila during space flight. Biol Sci Space 11: 346-350, 1997.

6. Ohnishi T, Inoue N, Matsumoto H, Omatsu T, Ohira Y and Nagaoka S: Cellular content of p53 protein in rat skin after exposure to the space environment. J Appl Physiol (1985) 81: 183-185, 1996.

7. Ohnishi T, Tsuji K, Ohmura T, Matsumoto H, Wang X, Takahashi A, Nagaoka S, Takabayashi A and Takahahsi A: Accumulation of stress protein 72 (hsp72) in muscle and spleen of goldfish taken into space. Adv Space Res 21: 1077-1080, 1998.

8. Farhood B, Goradel NH, Mortezaee K, Khanlarkhani N, Salehi E, Nashtaei MS, Mirtavoos-Mahyari H, Motevaseli E, Shabeeb D, Musa AE and Najafi M: Melatonin as an adjuvant in radiotherapy for radioprotection and radiosensitization. Clin Transl Oncol 21: 268-279, 2019.

9. Slominski AT, Hardeland R, Zmijewski MA, Slominski RM, Reiter RJ and Paus R: Melatonin: A cutaneous perspective on its production, metabolism, and functions. J Invest Dermatol 138: 490-499, 2018

10. Slominski AT, Kim TK, Kleszczyński K, Semak I, Janjetovic Z, Sweatman T, Skobowiat C, Steketee JD, Lin Z, Postlethwaite A, et al: Characterization of serotonin and $\mathrm{N}$-acetylserotonin systems in the human epidermis and skin cells. J Pineal Res 68: e12626, 2020.

11. Slominski RM, Reiter RJ, Schlabritz-Loutsevitch N, Ostrom RS and Slominski AT: Melatonin membrane receptors in peripheral tissues: Distribution and functions. Mol Cell Endocrinol 351: 152-166, 2012

12. Slominski AT, Zmijewski MA, Semak I, Kim TK, Janjetovic Z, Slominski RM and Zmijewski JW: Melatonin, mitochondria, and the skin. Cell Mol Life Sci 74: 3913-3925, 2017.

13. Tan DX, Manchester LC, Qin L and Reiter RJ: Melatonin: A mitochondrial targeting molecule involving mitochondrial protection and dynamics. Int J Mol Sci 17: 2124, 2016.

14. Zerbino DR and Birney E: Velvet: Algorithms for de novo short read assembly using de Bruijn graphs. Genome Res 18: 821-829, 2008.

15. Robertson G, Schein J, Chiu R, Corbett R, Field M, Jackman SD, Mungall K, Lee S, Okada HM, Qian JQ, et al: De novo assembly and analysis of RNA-seq data. Nat Methods 7: 909-912, 2010.

16. Xie Y, Wu G, Tang J, Luo R, Patterson J, Liu S, Huang W, He G, Gu S, Li S, et al: SOAPdenovo-Trans: De novo transcriptome assembly with short RNA-Seq reads. Bioinformatics 30: 1660-1666, 2014

17. Ikegame M, Hattori A, Tabata MJ, Kitamura KI, Tabuchi Y, Furusawa Y, Maruyama Y, Yamamoto T, Sekiguchi T, Matsuoka R, et al: Melatonin is a potential drug for the prevention of bone loss during space flight. J Pineal Res 67: e12594, 2019.

18. Yano S, Masuda D, Kasahara H, Omori K, Higashibata A, Asashima M, Ohnishi T, Yatagai F, Kamisaka S, Furusawa T, et al: Excellent thermal control ability of cell biology experiment facility (CBEF) for Ground-based experiments and experiments onboard the Kibo Japanese Experiment Module of International Space Station. Biol Sci Space 26: 12-20, 2012.

19. Bolger AM, Lohse $M$ and Usadel B: Trimmomatic: A flexible trimmer for Illumina sequence data. Bioinformatics 30 : 2114-2120, 2014

20. Smeds L and Kunstner A: ConDeTri-a content dependent read trimmer for Illumina data. PLoS One 6: e26314, 2011.

21. Haas BJ, Papanicolaou A, Yassour M, Grabherr M, Blood PD, Bowden J, Couger MB, Eccles D, Li B, Lieber M, et al: De novo transcript sequence reconstruction from RNA-seq using the Trinity platform for reference generation and analysis. Nat Protoc 8: 1494-1512, 2013.

22. Langmead B and Salzberg SL: Fast gapped-read alignment with Bowtie 2. Nat Methods 9: 357-359, 2012.
23. Robinson MD and Oshlack A: A scaling normalization method for differential expression analysis of RNA-seq data. Genome Biol 11: R25, 2010.

24. Huang da W, Sherman BT and Lempicki RA: Systematic and integrative analysis of large gene lists using DAVID bioinformatics resources. Nat Protoc 4: 44-57, 2009.

25. Jackson SP and Bartek J: The DNA-damage response in human biology and disease. Nature 461: 1071-1078, 2009

26. Winkler GS, Araujo SJ, Fieldler U, Vermeulen W, Coin F, Egly JM, Hoeijmakers JH, Wood RD, Timmers HT and Weeda G: TFIIH With Inactive XPD helicase functions in transcription initiation but is defective in DNA repair. J Biol Chem 275: 4258-4266, 2000

27. Hirao A, Kong YY, Matsuoka S, Wakeham A, Ruland J, Yoshida H, Liu D, Elledge SJ and Mak TW: DNA damage-induced activation of p53 by the checkpoint kinase Chk2. Science 287: $1824-1827,2000$

28. Shiloh Y and Ziv Y: The ATM protein kinase: Regulating the cellular response to genotoxic stress, and more. Nat Rev Mol Cell Biol 14: 197-210, 2013.

29. Hartwell LH and Kastan MB: Cell cycle control and cancer. Science 266: 1821-1828, 1994.

30. Amundson SA, Grace MB, McLeland CB, Epperly MW, Yeager A, Zhan Q, Greenberger JS and Fornace AJ Jr: Human in vivo radiation-induced biomarkers: Gene expression changes in radiotherapy patients. Cancer Res 64: 6368-6371, 2004.

31. Bouwman P and Jonkers J: The effects of deregulated DNA damage signalling on cancer chemotherapy response and resistance. Nat Rev Cancer 12: 587-598, 2012.

32. Takahashi A and Ohnishi T: Does gammaH2AX foci formation depend on the presence of DNA double strand breaks? Cancer Lett 229: 171-179, 2005.

33. van den Bosch M, Bree RT and Lowndes NF: The MRN complex: Coordinating and mediating the response to broken chromosomes. EMBO Rep 4: 844-849, 2003.

34. Mochan TA, Venere M, DiTullio RA Jr and Halazonetis TD: 53BP1 and NFBD1/MDC1-Nbs1 function in parallel interacting pathways activating ataxia-telangiectasia mutated (ATM) in response to DNA damage. Cancer Res 63: 8586-8591, 2003.

35. Lou Z, Minter-Dykhouse K, Wu X and Chen J: MDC1 is coupled to activated CHK2 in mammalian DNA damage response pathways. Nature 421: 957-961, 2003.

36. Yang Y, Gao Y, Zlatanou A, Tateishi S, Yurchenko V, Rogozin IB and Vaziri C: Diverse roles of RAD18 and Y-family DNA polymerases in tumorigenesis. Cell Cycle 17: 833-843, 2018.

37. Tanoue Y, Toyoda T, Sun J, Mustofa MK, Tateishi C, Endo S, Motoyama N, Araki K, Wu D, Okuno Y, et al: Differential roles of Rad18 and Chk2 in genome maintenance and skin carcinogenesis following UV Exposure. J Invest Dermatol 138: 2550-2557, 2018.

38. Reiter RJ, Tan DX, Manchester LC and Qi W: Biochemical reactivity of melatonin with reactive oxygen and nitrogen species: A review of the evidence. Cell Biochem Biophys 34: 237-256, 2001

39. Cui YF, Ding YQ, Zhang Y, Xu H, Jin W, Liu XL, Dong B, Mao JP and Mao BZ: Apoptotic characteristics of spleen lymphocyte in mice irradiated by lethal dose and its relationship to the expression of Bax and Bcl-XL proteins. Zhongguo Wei Zhong Bing Ji Jiu Yi Xue 17: 109-112, 2005 (In Chinese).

40. Mohseni M, Mihandoost E, Shirazi A, Sepehrizadeh Z, Bazzaz JT and Ghazi-khansari M: Melatonin may play a role in modulation of bax and bcl-2 expression levels to protect rat peripheral blood lymphocytes from gamma irradiation-induced apoptosis. Mutat Res 738-739: 19-27, 2012.

41. Garcia I, Cosio G, Lizarraga F, Martínez-Ruiz G, Meléndez-Zajgla J, Ceballos G, Espinosa M, Pacheco R and Maldonado V: Bcl-3 regulates UVB-induced apoptosis. Hum Cell 26: 47-55, 2013

42. Karanam NK, Grabarczyk P, Hammer E, Scharf C, Venz S, Gesell-Salazar M, Barthlen W, Przybylski GK, Schmidt CA and Völker U: Proteome analysis reveals new mechanisms of Bcl11b-loss driven apoptosis. J Proteome Res 9: 3799-3811, 2010.

43. Behl Y, Krothapalli P, Desta T, Roy S and Graves DT: FOXO1 plays an important role in enhanced microvascular cell apoptosis and microvascular cell loss in type 1 and type 2 diabetic rats. Diabetes 58: 917-925, 2009.

44. Browne G, Nesbitt H, Ming L, Stein GS, Lian JB, McKeown SR and Worthington $\mathrm{J}$ : Bicalutamide-induced hypoxia potentiates RUNX2-mediated Bcl-2 expression resulting in apoptosis resistance. Br J Cancer 107: 1714-1721, 2012.

45. Kato JY, Matsuoka M, Polyak K, Massagué J and Sherr CJ: Cyclic AMP-induced G1 phase arrest mediated by an inhibitor (p27Kip1) of cyclin-dependent kinase 4 activation. Cell 79: 487-496, 1994. 
46. Savell J, Rao S, Pledger WJ and Wharton W: Permanent growth arrest in irradiated human fibroblasts. Radiat Res 155: 554-563, 2001.

47. Juríková M, Danihel L, Polák Š and Varga II: Ki67, PCNA and MCM proteins: Markers of proliferation in the diagnosis of breast cancer. Acta Histochem 118: 544-552, 2016.

48. Baldwin J and Grantham V: Radiation Hormesis: Historical and current perspectives. J Nucl Med Technol 43: 242-246, 2015

49. Komori T: Regulation of proliferation, differentiation and functions of osteoblasts by Runx2. Int J Mol Sci 20: 1694, 2019.

50. Sakamaki J,Daitoku H, Yoshimochi K, Miwa M and Fukamizu A: Regulation of FOXO1-mediated transcription and cell proliferation by PARP-1. Biochem Biophys Res Commun 382: 497-502, 2009.

51. Malinen M, Saramäki A, Ropponen A, Degenhardt T, Väisänen S and Carlberg C: Distinct HDACs regulate the transcriptional response of human cyclin-dependent kinase inhibitor genes to Trichostatin A and 1alpha, 25-dihydroxyvitamin D3. Nucleic Acids Res 36: 121-132, 2008

52. Kawane T, Komori H, Liu W, Moriishi T, Miyazaki T, Mori M, Matsuo Y, Takada Y, Izumi S, Jiang Q, et al: Dlx5 and mef2 regulate a novel runx2 enhancer for osteoblast-specific expression. J Bone Miner Res 29: 1960-1969, 2014.

53. Naito J, Kaji H, Sowa H, Hendy GN, Sugimoto T and Chihara K: Menin suppresses osteoblast differentiation by antagonizing the AP-1 factor, JunD. J Biol Chem 280: 4785-4791, 2005.
54. Slominski AT, Zmijewski MA, Plonka PM, Szaflarski JP and Paus R: How UV light touches the brain and endocrine system through skin, and Why. Endocrinology 159: 1992-2007, 2018

55. Skobowiat C, Brozyna AA, Janjetovic Z, Jeayeng S, Oak ASW Kim TK, Panich U, Reiter RJ and Slominski AT: Melatonin and its derivatives counteract the ultraviolet $\mathrm{B}$ radiation-induced damage in human and porcine skin ex vivo. J Pineal Res 65: e12501, 2018

56. Janjetovic Z, Jarrett SG, Lee EF, Duprey C, Reiter RJ and Slominski AT: Melatonin and its metabolites protect human melanocytes against UVB-induced damage: Involvement of NRF2-mediated pathways. Sci Rep 7: 1274, 2017.

57. Kim TK, Kleszczynski K, Janjetovic Z, Sweatman T, Lin Z, Li W, Reiter RJ, Fischer TW and Slominski AT: Metabolism of melatonin and biological activity of intermediates of melatoninergic pathway in human skin cells. FASEB J 27: 2742-2755, 2013.

58. Slominski AT, Semak I, Fischer TW, Kim TK, Kleszczyński K, Hardeland R and Reiter RJ: Metabolism of melatonin in the skin: Why is it important? Exp Dermatol 26: 563-568, 2017.

This work is licensed under a Creative Commons Attribution-NonCommercial-NoDerivatives 4.0 International (CC BY-NC-ND 4.0) License. 